\title{
Attrition in emergency department point-of-care ultrasound workflow adherence for the evaluation of cutaneous abscesses
}

\author{
Stephen Alerhand ${ }^{1}$, Carl T. Mickman ${ }^{2}$, Kevin $\mathrm{Hu}^{2}$, Donald U. Apakama Jr², Jonathan M. Mishoe ${ }^{2}$, \\ Bret P. Nelson ${ }^{2}$ \\ ${ }^{1}$ Department of Emergency Medicine, Rutgers New Jersey Medical School, Newark, NJ, USA; ${ }^{2}$ Department of Emergency Medicine, Icahn School \\ of Medicine at Mount Sinai, New York, NY, USA \\ Contributions: (I) Conception and design: S Alerhand, CT Mickman, K Hu, BP Nelson; (II) Administrative support: None; (III) Provision of study \\ materials or patients: None; (IV) Collection and assembly of data: S Alerhand, CT Mickman, K Hu, DU Apakama Jr, JM Mishoe; (V) Data analysis \\ and interpretation: S Alerhand, BP Nelson; (VI) Manuscript writing: All authors; (VII) Final approval of manuscript: All authors. \\ Correspondence to: Stephen Alerhand, MD. 185 South Orange Avenue, Newark, NJ 07103, USA. Email: Stephen.Alerhand@gmail.com.
}

Background: Many emergency departments (ED) have implemented software solutions for ordering, documenting, and interpreting point-of-care ultrasound (POCUS) scans before healthcare bill generation. However, there are human and design barriers that prevent workflow completion. We sought to evaluate attrition in adherence to this step-wise workflow for evaluating cutaneous abscesses in a large urban ED, while quantifying missed potential revenue.

Methods: Patient charts in 2017 with discharge diagnoses containing "abscess", "boil”, or "cyst" were retrospectively extracted. Exclusion criteria included: POCUS not reasonably performed, abscess already draining, advanced imaging ordered, or consultant involvement. Each workflow step was assessed for completion. Revenue estimation was performed by multiplying number of scans by the appropriate relative value unit and medicare conversion factor.

Results: Of 2,240 total charts, 710 abscesses (31.7\%) met inclusion. Of those, 283 (39.8\%) POCUS were performed, of which 213 (30.0\%) were ordered, 198 (27.8\%) interpreted, and 180 (25.3\%) had images saved. Professional fees were billed for 120 POCUS examinations (16.9\%). There were 66 payments collected (9.3\%), amounting to $\$ 1,400.69$ revenue. Estimated billing for the 120 POCUS was $\$ 2,546.71$. If proper workflow had been implemented for all 283 POCUS performed, estimated revenue would have been $\$ 6,006.00$. If POCUS had been performed with proper workflow for all 710 abscesses, estimated revenue would have been $\$ 15,068.05$.

Conclusions: POCUS workflow was interrupted at several points and completed sub-optimally. This attrition directly affected optimal patient care, documentation, and departmental revenue. Since cutaneous abscesses represent one of many ED POCUS applications, the extrapolated missed potential revenue would be much greater overall.

Keywords: Point-of-care ultrasound (POCUS); workflow; abscess

Received: 14 June 2020; Accepted: 04 November 2020; Published: 25 December 2020.

doi: 10.21037/jhmhp-20-85

View this article at: http://dx.doi.org/10.21037/jhmhp-20-85

\footnotetext{
^ ORCID: 0000-0003-2383-0309.
} 


\section{Introduction}

Evidence supports the use of point-of-care ultrasound (POCUS) for evaluating skin and soft tissue infections (SSTI's) in the emergency department (ED). To begin with, its sensitivity, specificity, positive predictive value, and negative predictive value for detecting an abscess are all greater than using clinical exam alone (1-8). The use of POCUS for evaluating SSTI's may also decrease ED length-of-stay (LOS) compared to ultrasounds performed by radiology technicians (9). POCUS may also change management $(5,6,10,11)$, especially when the diagnosis is uncertain (12). Additionally, its incorporation is associated with a decreased likelihood of failing therapy and requiring repeat incision and drainage (I\&D) $(13,14)$. POCUS may thus increase confidence in performing the procedure (10). Lastly, the evaluation of SSTI's with POCUS can be learned by novice sonographers with only minimal training and shows good interrater reliability compared to experts $(3,15)$, whereas clinical exam alone shows only fair-tomoderate inter-examiner agreement $(16,17)$. Therefore, we chose this particular POCUS modality to assess adherence to an expected workflow of POCUS performance, documentation, and billing.

Many EDs have implemented electronic medical record (EMR) workflow solutions for properly ordering, documenting, and interpreting POCUS scans before ultimately producing a bill for healthcare revenue (18-21). This practice is supported by guidelines from the American College of Emergency Physicians (ACEP) (22). However, many departments struggle with clinicians appropriately documenting POCUS findings, with resultant poor reimbursement for those scans performed. When examining a POCUS workflow, there are many possible places where cases can be lost: incomplete documentation of the scan, documentation not completed in real-time, ultrasound machine maintenance, delays in wireless transmission of the scan to the EMR, short EMR logout times, provider awareness of the workflow process, and attending physician compliance (23). Thus, it is important to break down the workflow into parts.

In this retrospective descriptive study, we describe our experience examining our own workflow for areas where we would improve throughput and compliance with departmental guidelines: patient selection, order entry, image archival, documentation of findings, billing, and reimbursement. Specifically, we sought to evaluate the attrition rate in our step-wise POCUS workflow for evaluating cutaneous abscesses in a large urban ED over one year. Based on our findings, we hoped to determine which aspects of the workflow could be improved to increase compliance in this and other POCUS applications in patient care. We also attempted to quantify the potential missed revenue from lost charges on medical care that was provided.

We present the following article in accordance with the MDAR checklist (available at http://dx.doi.org/10.21037/ jhmhp-20-85).

\section{Methods}

\section{Study setting}

A retrospective chart review was performed for the 2017 calendar year in an urban, academic medical center with an emergency medicine (EM) residency and emergency ultrasound (EUS) fellowship. The study was deemed exempt from the hospital's Institutional Review Board.

\section{Data extraction}

Medical charts with discharge diagnoses containing the words "abscess", "boil", or "cyst" were initially selected for screening from the EMR by the ED Director of Informatics. Data extraction from these charts was divided up equally and performed by EUS Fellows (SA, KU, CTM), as well as by the EM residents (DUA, JMM) on their scheduled POCUS Rotation at the time of the study's conception. All data extractors had undergone formal hospital training in the use of the EMR (Epic, Verona, Wisconsin). The data extraction form was created on Microsoft Excel.

\section{Exclusion criteria}

Charts were excluded in advance for the following conditions: (I) POCUS could not be performed or was not reasonably indicated due to inaccessible anatomic location (i.e. intra-abdominal abscess, dental abscess). (II) The diagnosis of abscess was clear from the outset, as per medical provider documentation describing an abscess already draining significantly. (III) Advanced imaging such as computed tomography (CT) or magnetic resonance imaging (MRI) was ordered, rendering a POCUS scan redundant. (IV) A consultant was involved in the medical decision-making, and thus, the decision to scan or drain the SSTI may not have entirely been the EM physician's 


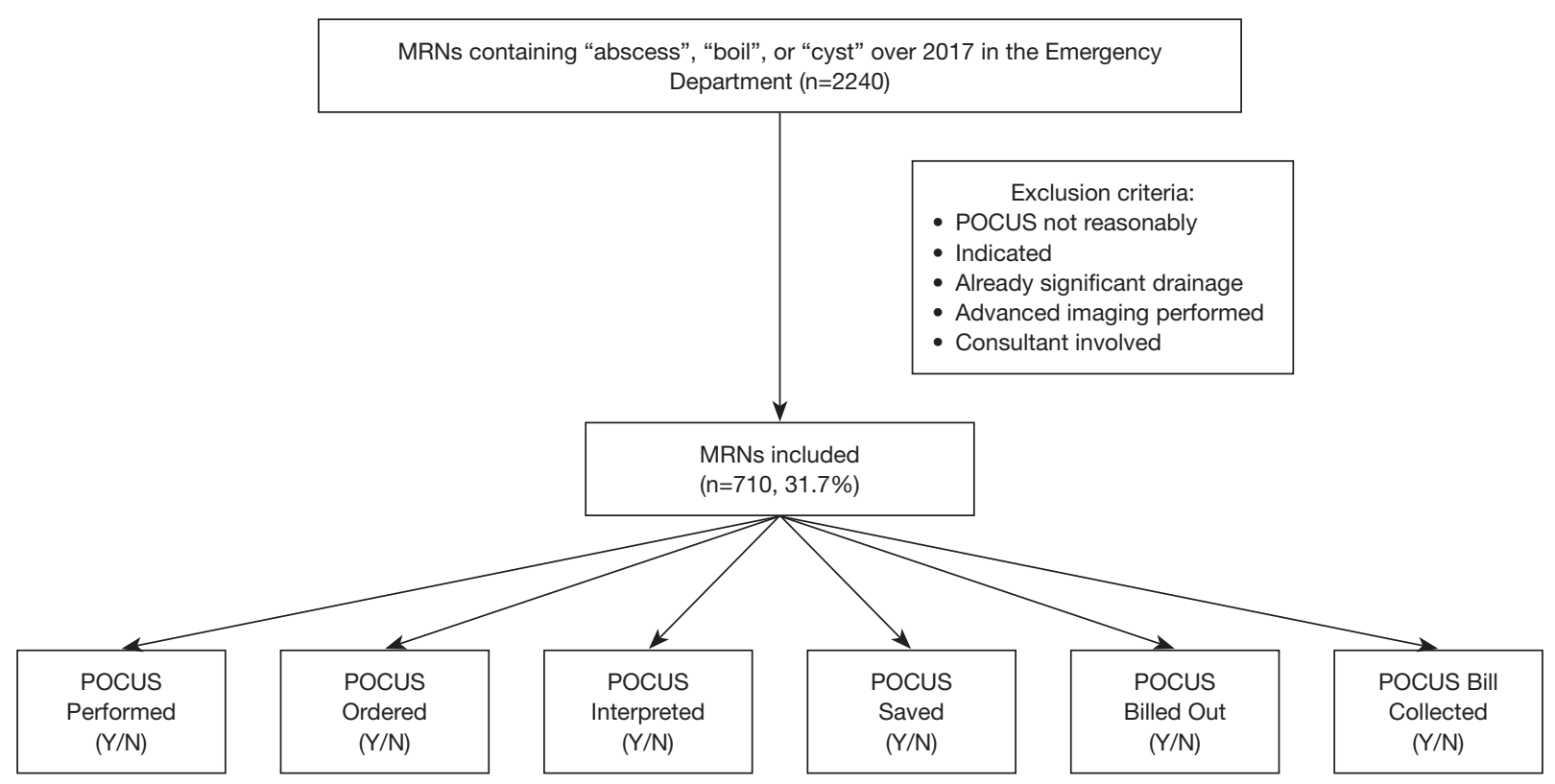

Figure 1 Methodology flow chart.

decision (Figure 1). Any charts indeterminate for exclusion were referred to the study leaders (SA, BPN) for final determination.

\section{EUS workflow}

The workflow for ordering and documenting POCUS scans went according to the following manner: (I) The physician orders the POCUS scan in the EMR. (II) The physician accesses the patient's POCUS scan on the ultrasound machine by both the patient's name and medical record number (MRN) (listed on wristband upon entry to ED). (III) The physician performs the scan, then saves images and clips correlating to the patient's medical record number. (IV) On completion of the scan, the ultrasound machine transfers the patient information and saved media files to the ultrasound image archive (SoftLink International, White Plains, NY, USA). (V) The attending physician creates a Procedure Note in the EMR for interpreting the POCUS findings to indicate "abscess" or "no abscess", along with other descriptors as needed. (VI) The hospital coders send the POCUS scan bill to the payer. (VII) The hospital collects the revenue for this bill.

\section{EMR outcome measures}

The following workflow data points were extracted for each patient chart: was a POCUS scan performed? Was it ordered? Was it interpreted in the EMR? Were images or clips saved to the ultrasound image archive? Was the POCUS procedure billed to the payer? Was the revenue collected? No advanced statistical analysis was required.

\section{Billing and revenue outcome measures}

Whenever physician groups discuss fees or payments for services, there is a risk that data could be used inappropriately or misconstrued as a component of price fixing or restraint of trade. Therefore, we have used publicly available payment data to avoid this possibility, as well as to provide the most generalizable information for providers around the United States. Estimation of billed and collected revenue was performed by multiplying the number of POCUS by the "soft tissue ultrasound" relative value unit (RVU) and the 2017 Medicare Conversion Factor. The "soft tissue ultrasound" RVU designation was found in the 2017 National Medicare Physician Fee Schedule (MPFS), as described in the ACEP Ultrasound Section Coding and Reimbursement Update (24). "Soft tissue ultrasound" is broken up into separate current procedural terminology (CPT) codes for various body locations, each with slightly varied RVU amounts. For our calculations, we used the average of the RVU amounts specifically referring to cutaneous abscesses. 
Table 1 Demographic and ancillary information

\begin{tabular}{ll}
\hline Variable & Outcome \\
\hline Median age in years (IQR) & $24.5(19.8,34.0)$ \\
Male gender & $411(58.8 \%)$ \\
I\&D performed & $614(86.5 \%)$ \\
\hline
\end{tabular}

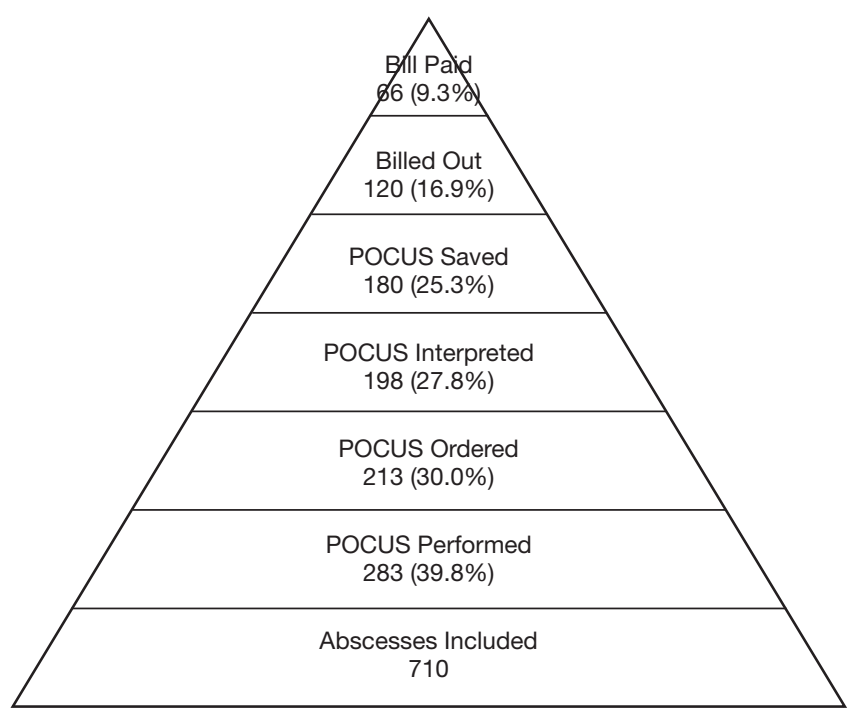

Figure 2 Attrition of the point-of-care ultrasound workflow for evaluating cutaneous abscesses.

\section{Results}

Out of 2,240 total charts extracted, there were 710 abscesses $(31.7 \%)$ that met inclusion criteria (Figure 1). For these patients, the median age in years was 24.5 years $(19.8,34.0)$, gender was male in $411(57.8 \%)$, and I\&D was performed in 614 (86.5\%) (Table 1). In the workflow, 283 (39.8\%) POCUS were performed, $213(30.0 \%)$ were ordered in the EMR, $198(27.8 \%)$ were interpreted in the EMR, and $180(25.3 \%)$ were saved in the ultrasound image archive (Figure 2). There were 120 POCUS billed (16.9\%). Of these, there were 66 payments collected $(9.3 \%)$.

The average "soft tissue ultrasound" RVU for the separate CPT codes in the 2017 MPFS was 0.56. The 2017 Medicare Conversion Factor was 37.8975. Based on these figures, the 66 payments collected would amount to a revenue of $\$ 1,400.69$ (Figure 3). The billed amount for the 120 POCUS would have been $\$ 2,546.71$. If the proper workflow and bill collection had been implemented for all 283 POCUS performed, the revenue would have been $\$ 6,006.00$. If POCUS had been performed with the proper workflow and bill collection for all 710 abscesses, the maximal potential revenue would have been $\$ 15,068.05$. These numbers do not reflect potential facility fees which can be charged by the hospital for the performance of POCUS examinations.

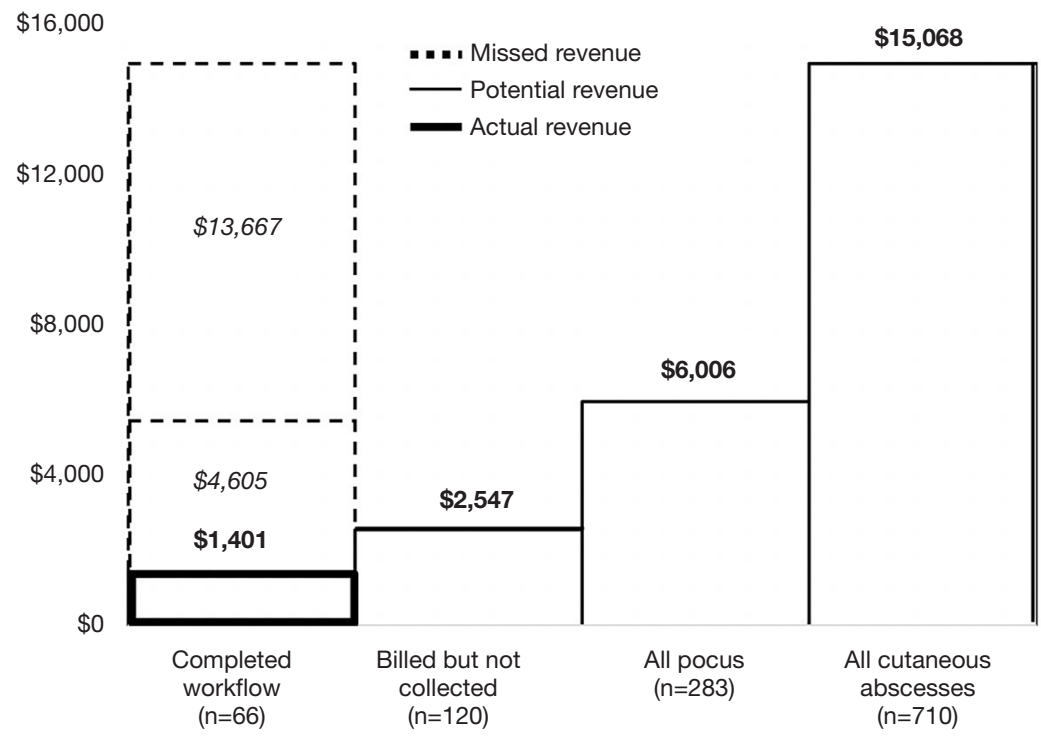

Figure 3 Professional fee revenue collection based on point-of-care ultrasound scans for cutaneous abscesses. 


\section{Discussion}

In the step-wise process of completing the POCUS workflow, our retrospective descriptive study found that the workflow was interrupted at various points. To begin with, a POCUS scan to evaluate the SSTI may not have even been performed. Additionally, the emergency physician may not have ordered the scan before performing it and completing the EMR interpretation. The physician may also not have saved the images and clips into the ultrasound image archive. Furthermore, even if the physician completed their role, the hospital billing department may not have sent out the bill, and if sent, the insurance company or other payer may not have delivered the revenue.

In simplest terms, for every 100 abscess cases, 87 I\&D procedures were performed. In 40 cases, POCUS was performed, 28 of which were properly interpreted, and 25 of which had images saved. Only 17 were billed, and 9 of those were paid. By understanding the attrition through all steps, we can learn where best to focus our energies in improving patient care. Should we be assessing more patients with POCUS prior to the decision to $I \& D$, as the literature would suggest? In our data set, sixty percent of potential abscess cases did not have an ultrasound. This could represent practice variation, interpretation of the literature, availability of machines, time pressure, or a host of other factors. Of those POCUS exams that were performed, there were many missed opportunities in image saving and documentation. This could represent time pressure, challenges with using software, education, and other factors. Finally, significant attrition in billing and payments could be due to coder education or denial of charges. By using this simple model based on abscess evaluation, we can examine our system to improve a variety of different types of ultrasounds and patient care models.

We elected to review the ED's workflow adherence for potential cutaneous abscesses, because it can reasonably be inferred from the evidence that SSTI's would benefit from evaluation with POCUS. There are at least three ways POCUS can change management of SSTI's: (I) What seems to be a simple abscess based on clinical exam is actually loculated, deeper, or otherwise more complex when examined with POCUS. Rather than a simple I\&D, further imaging or a Surgical consultation may be indicated. (II) What looks like an abscess based on clinical exam turns out to have no evident fluid collection, so no I\&D is performed. (III) What appears to be cellulitis only based on clinical exam is revealed on POCUS to be an abscess requiring drainage.

From our initial chart review, we excluded cases in which POCUS could not be performed such as for dental, intraabdominal, or other non-cutaneous abscesses. We also excluded cases in which failure to perform POCUS could reasonably be understood. This included cases in which the abscess was already draining significantly or when advanced imaging such as CT or MRI was performed. Lastly, we excluded cases in which a consultant was involved early in the medical decision-making and thus, the decision to perform a POCUS may not have been directly determined by the ED team. This ED is affiliated with a tertiary care hospital where patients with complicated existing medical problems may have specialist care.

In this ED, the reasons for the POCUS workflow attrition rate were likely similar to those described by a workflow task force that evaluated their ED's POCUS documentation and billing (23). To begin with, time and workload constraints may have precluded a resident or attending from ordering the POCUS study in the EMR. The attending physician may also have neglected to write an interpretation "Procedure Note". This ED treats over 130,000 patient visits per year, and a prior study found that EM physicians have 9.7 workflow interruptions per hour (25). Additionally, it is possible that the machine's image-saving mechanism or wireless transmission to the image archive could have experienced an interruption in service. This information could not be captured retrospectively. Furthermore, physicians in this ED had been instructed on the proper POCUS workflow, but the possibility remains that more frequent tutorials would be beneficial.

Some potential solutions addressed by the aforementioned workflow task force may also be applicable in this ED (23). One intervention would be to initiate automated inbox deficiency reports for all "soft tissue ultrasounds" entered into the EMR. Just like many EMR's or billing departments notify physicians of outstanding unsigned charts, the same could be done specifically for POCUS scans. Additionally, the workflow process could be periodically reviewed at faculty meetings and resident conferences. Reminder signs at ED provider stations would also be helpful. Furthermore, education on the use of POCUS for evaluating SSTI's could be provided for both attendings and residents alike. Other interventions include EMR charting templates for ease of documentation, barcode scanners to rapidly pull up a particular patient's scan, and the use of scribes. Along with a positive effect on patient care and safety, the effect of such interventions would presumably be greater departmental 
revenue for services which are already being provided.

The attrition rate in POCUS workflow adherence directly affected departmental revenue. Of the 283 POCUS scans performed for evaluating cutaneous abscesses in the 2017 calendar year, only 66 proceeded through the entire workflow-from ordering of the scan in the EMR all the way to revenue collection. This amounted to only $\$ 1,400.69$ in collections as modelled by our revenue simulation. If the workflow had been followed for all 283 POCUS performed, the revenue would have been $\$ 6,006.00$. Thus, only $23.32 \%$ of potential revenue was collected for services already performed, with a missed potential revenue of $\$ 4,605.31$. Theoretically, if the workflow had been completed for all 710 cutaneous abscesses, the maximal potential revenue would have been $\$ 15,068.05$. This amounts to a missed potential revenue of $\$ 13,667.36$ over the calendar year. SSTI's are just one of many common POCUS applications commonly used in the ED (22), so the missed potential revenue across all POCUS applications would be much greater overall.

Moreover, the revenue discussed only reflects professional fee revenue, not facility fees charged by the hospital. Professional fees for POCUS procedures are often directly billed to payers as well as charged regardless of patient disposition. However, the corresponding facility charges are much more likely to be bundled into global emergency visit payments and global hospital charges for admitted patients. These and other factors make delineating facility charges for ultrasound procedures quite challenging and hard to reliably quantify. For these reasons, we did not quantify the impact of facility charges in this data set.

There are few other descriptions of POCUS workflow processes in the literature, but systems and operations have seemingly improved with time. To begin with, based on a survey completed by residency and ultrasound directors in 2003, only $16 \%$ of EM programs reported billing for POCUS scans (21). However, $12 \%$ planned to bill within one year, and $37 \%$ planned to bill at some point in the future. In 2009, a retrospective analysis of ED POCUS found that an ED that bills for trauma and procedural US and achieves its potential billing volume would break even in less than 5 years (18). By 2014, a retrospective review sought to determine the fiscal impact of introducing the Q-Path ultrasound archiving software for POCUS billing and reimbursement (19). There was an increase in ED faculty participation in billing from $30 \%$ to $75 \%$ after Q-Path implementation. There was also an increase in POCUS exams billed from 857 to 4,449 . Facility fee (reported by the hospital) revenue increased seven-fold, and professional fee (reported and generated by the physician for services provided) revenue increased 6.34-fold (19).

In 2016, a workflow task force sought to assess the effect of direct education and personal feedback on adherence to POCUS documentation and workflow (23). The authors found that $41 \%$ of scans were documented correctly before the task force's creation compared to $63 \%$ afterwards, out of a similar number of scans performed. They also found that $15.9 \%$ of scans were billed prior to the intervention compared to $32 \%$ afterwards. A subsequent 2017 study took these operations further by evaluating the impact of a more automated system on POCUS billing (20). In this system, both patient and physician wore barcode wristbands that were scanned at the bedside to access the proper study on the ultrasound machine. Q-Path documentation was completed at the bedside after image or clip acquisition. The authors found a statistically significant increase in the number of scans billed, along with an increase in the percent of scans billed for both the facility (32\% to $61 \%$ ) and professional (37\% to $65 \%$ ) billing components. Accordingly, there was a $96 \%$ and $78 \%$ increase in revenue for facility and professional fees, respectively.

As a retrospective study, ours inherently carried some selection bias. However, we sought to limit this effect by including all patients with a final EMR diagnosis containing "abscess", "boil", or "cyst", and then manually reviewing each of those charts.

Our descriptive study may not be generalizable to all institutions for two reasons: (I) The POCUS workflow used may vary from that in other ED's. (II) The EDs payer mix determines reimbursement for physician services, and this varies between institutions as well. However, by using the standard RVUs and the 2017 Medicare Conversion Factor, the estimated revenue amounts may be approximately extrapolated to other EDs. The exact workflow for ultrasound image ordering, acquisition, storage, and billing may vary from institution to institution. However, similar processes (such as ordering and interpreting the POCUS scan in the EMR or ultrasound image archive) will be found across the United States, and similar obstacles may be faced at each step. Therefore, we hope that our experience can help other institutions assess the critical steps in their workflow, establish benchmarks and goals, and track their own metrics as they seek to streamline the process and increase compliance. 


\section{Conclusions}

Evidence supports the incorporation of POCUS into the evaluation of SSTI's. This descriptive study depicts the step-wise attrition in adherence to the POCUS workflow for the performance, documentation, and billing of this service in the ED. It shows the different points at which the workflow can be interrupted and therefore optimized, i.e. more POCUS scans could have been performed, ordered properly, documented properly, and had images saved. It also posits potential explanations for this suboptimal adherence to protocol. By using standard RVU's and the 2017 Medicare Conversion Factor, this study calculates the potential healthcare revenue that is not being collected for physician services already being performed. Considering the varied POCUS applications used in the ED, the missed potential revenue extrapolated to all applications would be much greater overall. This POCUS documentation and billing information may be valuable for Ultrasound Division Directors, ED Chairs, and hospital administrators.

\section{Acknowledgments}

Funding: None.

\section{Footnote}

Reporting Checklist: The authors have completed the MDAR checklist. Available at http://dx.doi.org/10.21037/ jhmhp-20-85

Data Sharing Statement: Available at http://dx.doi. org/10.21037/jhmhp-20-85

Conflicts of Interest: All authors have completed the ICMJE uniform disclosure form (available at http://dx.doi. org/10.21037/jhmhp-20-85). BPN reports that he is on the Medical Advisory Boards for DiA and Echonous, and is a consultant for General Electric. SA and $\mathrm{KH}$ were the Clinical Ultrasound Fellows, CTM was the incoming Clinical Ultrasound Fellow, and DUA and JMM were the residents on the ultrasound rotation at the time of commencing the study. BPN is the Ultrasound Division Director.

Ethical Statement: The authors are accountable for all aspects of the work in ensuring that questions related to the accuracy or integrity of any part of the work are appropriately investigated and resolved. The study was conducted in accordance with the Declaration of Helsinki (as revised in 2013). The study was deemed exempt from the hospital's Institutional Review Board.

Open Access Statement: This is an Open Access article distributed in accordance with the Creative Commons Attribution-NonCommercial-NoDerivs 4.0 International License (CC BY-NC-ND 4.0), which permits the noncommercial replication and distribution of the article with the strict proviso that no changes or edits are made and the original work is properly cited (including links to both the formal publication through the relevant DOI and the license). See: https://creativecommons.org/licenses/by-nc-nd/4.0/.

\section{References}

1. Subramaniam S, Bober J, Chao J, et al. Point-of-care Ultrasound for Diagnosis of Abscess in Skin and Soft Tissue Infections. Acad Emerg Med 2016;23:1298-306.

2. Barbic D, Chenkin J, Cho DD, et al. In patients presenting to the emergency department with skin and soft tissue infections what is the diagnostic accuracy of point-of-care ultrasonography for the diagnosis of abscess compared to the current standard of care? A systematic review and meta-analysis. BMJ Open 2017;7:e013688.

3. Berger T, Garrido F, Green J, et al. Bedside ultrasound performed by novices for the detection of abscess in ED patients with soft tissue infections. Am J Emerg Med 2012;30:1569-73.

4. Squire BT, Fox JC, Anderson C. ABSCESS: applied bedside sonography for convenient evaluation of superficial soft tissue infections. Acad Emerg Med 2005;12:601-6.

5. Adams CM, Neuman MI, Levy JA. Point-of-Care Ultrasonography for the Diagnosis of Pediatric Soft Tissue Infection. J Pediatr 2016;169:122-7.e1.

6. Iverson K, Haritos D, Thomas R, et al. The effect of bedside ultrasound on diagnosis and management of soft tissue infections in a pediatric ED. Am J Emerg Med 2012;30:1347-51.

7. Ramirez-Schrempp D, Dorfman DH, Baker WE, et al. Ultrasound soft-tissue applications in the pediatric emergency department: to drain or not to drain? Pediatr Emerg Care 2009;25:44-8.

8. Marin JR, Dean AJ, Bilker WB, et al. Emergency ultrasound-assisted examination of skin and soft tissue infections in the pediatric emergency department. Acad 
Emerg Med 2013;20:545-53.

9. Lin MJ, Neuman M, Rempell R, et al. Point-of-Care Ultrasound is Associated With Decreased Length of Stay in Children Presenting to the Emergency Department With Soft Tissue Infection. J Emerg Med 2018;54:96-101.

10. Greenlund LJS, Merry SP, Thacher TD, et al. Primary Care Management of Skin Abscesses Guided by Ultrasound. Am J Med 2017;130:e191-3.

11. Tayal VS, Hasan N, Norton HJ, et al. The effect of softtissue ultrasound on the management of cellulitis in the emergency department. Acad Emerg Med 2006;13:384-8.

12. Mower WR, Crisp JG, Krishnadasan A, et al. Effect of Initial Bedside Ultrasonography on Emergency Department Skin and Soft Tissue Infection Management. Ann Emerg Med 2019;74:372-80.

13. Gaspari RJ, Sanseverino A, Gleeson T. Abscess Incision and Drainage With or Without Ultrasonography: A Randomized Controlled Trial. Ann Emerg Med 2019;73:1-7.

14. Gaspari RJ, Sanseverino A. Ultrasound-Guided Drainage for Pediatric Soft Tissue Abscesses Decreases Clinical Failure Rates Compared to Drainage Without Ultrasound: A Retrospective Study. J Ultrasound Med 2018;37:131-6.

15. Marin JR, Alpern ER, Panebianco NL, et al. Assessment of a training curriculum for emergency ultrasound for pediatric soft tissue infections. Acad Emerg Med 2011;18:174-82.

16. Giovanni JE, Dowd MD, Kennedy C, et al. Interexaminer agreement in physical examination for children with suspected soft tissue abscesses. Pediatr Emerg Care 2011;27:475-8.

17. Marin JR, Bilker W, Lautenbach E, et al. Reliability of

doi: $10.21037 /$ /hmhp-20-85

Cite this article as: Alerhand S, Mickman CT, Hu K, Apakama DU Jr, Mishoe JM, Nelson BP. Attrition in emergency department point-of-care ultrasound workflow adherence for the evaluation of cutaneous abscesses. J Hosp Manag Health Policy 2020;4:36. clinical examinations for pediatric skin and soft-tissue infections. Pediatrics 2010;126:925-30.

18. Soremekun OA, Noble VE, Liteplo AS, et al. Financial impact of emergency department ultrasound. Acad Emerg Med 2009;16:674-80.

19. Adhikari S, Amini R, Stolz L, et al. Implementation of a novel point-of-care ultrasound billing and reimbursement program: fiscal impact. Am J Emerg Med 2014;32:592-5.

20. Flannigan MJ, Adhikari S. Point-of-Care Ultrasound Work Flow Innovation: Impact on Documentation and Billing. J Ultrasound Med 2017;36:2467-74.

21. Moore CL, Gregg S, Lambert M. Performance, training, quality assurance, and reimbursement of emergency physician-performed ultrasonography at academic medical centers. J Ultrasound Med 2004;23:459-66.

22. Ultrasound Guidelines: Emergency, Point-of-Care and Clinical Ultrasound Guidelines in Medicine. Ann Emerg Med 2017;69:e27-54.

23. Lewiss RE, Cook J, Sauler A, et al. A workflow task force affects emergency physician compliance for point-of-care ultrasound documentation and billing. Crit Ultrasound J 2016;8:5.

24. Emergency Ultrasound Coding Guide 2016. In: American College of Emergency Physicians - Ultrasound Section. Available online: https://www.acep.org/globalassets/ uploads/uploaded-files/acep/membership/sections-ofmembership/ultra/acep-us-cpt-update-2016.pdf

25. Chisholm CD, Dornfeld AM, Nelson DR, et al. Work interrupted: a comparison of workplace interruptions in emergency departments and primary care offices. Ann Emerg Med 2001;38:146-51. 